\title{
Training effects on motor-cognitive dual-task performance in older adults
}

\author{
A systematic review
}

\author{
Bettina Wollesen • Claudia Voelcker-Rehage
}

Received: 1 July 2012 / Accepted: 6 February 2013 /Published online: 24 February 2013

(C) European Group for Research into Elderly and Physical Activity (EGREPA) 2013

\begin{abstract}
This systematic review investigated whether healthy older adults benefit from training interventions in motor-cognitive dual-task (DT) situations and which specific aspects of the intervention and/or task selection contribute to training benefits. Training effects were analysed with regard to the training programme (e.g., general ST or DT training) and task conditions (e.g., standing or walking, complexity of secondary cognitive task). Literature was searched via OVIDsp (Medline, EMBASE, PsycINFO). DT studies were included by the following criteria: (1) investigation of at least one motor task, (2) assessment of DT performance outcomes on standing or walking, (3) conduction of an intervention, and (4) investigation of older adults in an experimental-control group design or an old-young comparison. Thirteen studies met all inclusion criteria. Four types of interventions were identified: (1) general single-task (ST) motor training, (2) specific ST motor training, (3) general DT training, and (4) task-related (specific) DT training. For DT standing conditions only DT interventions improved motor performance, whereas DT walking also benefits by ST training. Most benefits on motor and cognitive performance seem to be reached by DT training interventions whereas a GST produced lowest effects. Thus, balance orientated motor and cognitive DT performance in healthy older adults can be improved by performance related exercises. Furthermore, to reach beneficial effects, it seems necessary that the training intervention includes a
\end{abstract}

\footnotetext{
B. Wollesen $(\triangle)$

Department of Human Movement Science, University of

Hamburg, Mollerstr. 2,

20148, Hamburg, Germany

e-mail: bettina.wollesen@uni-hamburg.de

C. Voelcker-Rehage

Jacobs Center on Lifelong Learning and Institutional

Development, Jacobs University, Bremen, Germany
}

certain level of exercise load such as rising difficulties, appropriate intensity and duration, a certain level of task specificity, and variable task prioritization. The transfer of training effects into everyday situations needs to be further investigated.

Keywords Ageing $\cdot$ Dual-task performance $\cdot$ Standing . Walking $\cdot$ Intervention $\cdot$ Motor control $\cdot$ Motor-cognitive tasks

\section{Introduction}

Most daily activities require the management of motorcognitive tasks while simultaneously processing external information: for example, crossing a street while observing traffic flow or thinking about the shopping list while carrying a cup of tea from one room to another. These motorcognitive interactions can be described as motor-cognitive dual-task (DT) performance. Studies have shown that older adults perform more poorly than younger adults when simultaneously engaged in cognitive and balance or walking tasks simultaneously [95]. In daily life situations this is, for example, associated with an increased fall risk $[2,20]$.

DT paradigms have been used to examine age-related changes in motor-cognitive performance and cognitive capacities. Motor and cognitive performance declines in DT situations are considered in light of several theoretical positions: The central bottleneck theory states that due to an information processing bottleneck only one task can be processed at a time; processing of a second task cannot commence until the first is complete. This bottleneck usually results in a longer response time for one of the two tasks within a DT paradigm [52, 53, 92]. The four-dimensional multiple resource model [95] proposes that there will be greater interference between two tasks to the extent that they 
share stages, sensory modalities, processing codes, and channels of visual information. Finally, the attentional resource theory suggests that declines in motor-cognitive functioning under DT conditions result from interference caused by competing demands for attentional resources. This results in less attention available to each task [32]. Motor-cognitive functioning is expected to deteriorate in complex situations if there are fewer resources available for performance than are required. Theories differ in whether the resources are assumed to be a single, general-purpose unit or structure [32] or whether multiple resources are assumed, such as modalities of stimulus input or response modes [48, 94].

Age is assumed to be associated with reduced processing efficiency (e.g., nerve conduction speed, fluid intelligence) $[27,33]$. Additionally, it is proposed that motor aspects of performance, like walking, are increasingly in need of cognitive control and supervision with advancing age [4, 38]. For example, it has been shown that gait and balance, as in seemingly simple tasks like routine walking, are rather complex motor tasks [25] and require a large amount of higher-level cognitive input [103]. Proposed mechanisms that necessitate the need for greater cognitive control include sensory losses, impaired or less automated motor and cognitive performance, and declines in the efficiency of cognitive control $[4,38]$. The reduced capacity hypothesis suggests that a motor task exerts a higher demand on the attentional resources of older than of younger adults to achieve the same performance level. Due to this, DT costs appear to be greater in older than in young adults [1]. In a review of meta-analyses and a meta-analysis on cognitive DT performance Verhaeghen and co-workers [87] concluded that older adults' processing costs emerge under DT conditions and that these age-associated deficits in cognitive DT performance exist over and beyond the effect of general age-related cognitive slowing [88].

Besides the higher-level of cognitive input required by gait and balance [103], walking in older adults is generally characterized by a lower velocity, cadence, stride length, and swing phase and standing is characterized with increased postural sway thus seems to be more difficult for older as compared to younger adults [37]. For motor-cognitive DT performance while standing or walking results are heterogeneous. Whereas, some study results revealed similar to the cognitive DT studies - that the concurrent performance of a static or dynamic balance tasks (i.e., standing or walking) and a cognitive task leads to increased postural sway and reduced stability while standing or walking, i.e., DT costs increase with increasing task demands $[26,73,96]$, more and more research results illustrated that the effects of a DT situation on motor outcomes are specific. One typical finding is that under certain DT conditions, involving a standing or walking task and a visual cognitive task, postural sway decreases and stability increases. For example, Stoffregen et al. [74, 75] revealed that during the concurrent performance of a balance task and a visual observation task postural sway is reduced indicating that postural stability is necessary to manage the secondary task, i.e., focus on the observation object (e.g., [41]). Other findings are that balance performance is improved in the most challenging DT situations (e.g., $[17,76])$ and that the size of the observed age effects in DT situations is modulated by task similarity or task complexity, respectively $[5,8,26]$, and temporal constraints $[42,88]$. Furthermore, task prioritization seems to be an important performance factor within motor-cognitive DT studies. In a literature review, Schäfer and Schumacher (cf. [58]) pointed out that older adults show a tendency to prioritize gait performance over the cognitive task, which might be explained by protecting oneself from falls [58]. Furthermore, a significant association between changes in DT performance and an increased fall risk in older adults has been shown, especially in frail older adults and adults with a history of falls $[5,70,72]$. That is, older adults with a history of falls performed more inefficient in DT situations than healthy older or younger adults, indicating that fallers might have problems to shift attention in DT situations and in turn to prioritize gait [58].

All these findings suggest that motor-cognitive performance changes in gait and balance control under DT conditions are a result of task settings, selection of motor and secondary cognitive task, study designs and examined target groups. Schäfer and Schumacher [58] proposed an inverted U-shape relationship between the efficacy of postural control and concurrent cognitive demands suggesting that focusing exclusively on a simple motor task like standing (which is usually performed automatically) leads to performance decrements, whereas a certain amount of cognitive load (secondary cognitive task) leads to improved motor performance, e.g., postural stability. When the cognitive load is further increased motor performance again might decline.

Over the last decades, it has been shown that systematic motor and cognitive training can improve older adults' cognitive and motor performance and reduce the number of disabilities [11, 51, 69]. For example, balance and coordination training as well as Tai Chi seem to gain high benefits for fall prevention (for a review, see [21, 61]); and the precision of task managing and attentional allocation stays at a high level in older adults when they were given adequate practice or feedback [7, 54].

Also improvements in motor-cognitive DT performance through training and practice have been investigated. Most DT training studies examined the effects of cognitive training on cognitive DT performance in older and younger adults $[7,8,81]$ using different cognitive ST or DT settings [54]. The number of motor-cognitive DT training studies, 
however, also increased during the last years. One key assumption of motor-cognitive DT training is that it frees up cognitive resources that were previously used to monitoring motor and/or cognitive performance [7]. Thus, older adults are able to use these resources to improve their motor and/or cognitive performance under DT conditions. Whereas earlier studies mainly focused on motor-cognitive driving tasks (e.g., [81]), recent studies start to investigate DT training paradigms by the use of standing and walking tasks with a special focus on fall prevention [65]. In a first review of Pichierri and colleagues [56], cognitive and motor-cognitive DT interventions which aimed to improve physical functioning in healthy or mild cognitive impaired older adults were summarized. The review cross-referenced to only seven DT interventions [62, 63, 65-67, 82, 104] showing positive effects of DT interventions on physical functioning. Following Pichierri et al. [56], the results of the included DT studies did not allow defining a training methodology with greatest effectiveness to improve physical functioning. Moreover the outcome results did not explicitly focus on functional improvements in motor-cognitive DT situations (two of the DT studies did not report DT performance as an outcome measurement $[62,63]$ or the influence of the task characteristics of the cognitive task on motor-cognitive DT performance. A review by SegevJacubovski and colleagues [60] summarized studies that evaluated the effects of cognitive therapy on fall risks referring to eight motor-cognitive $[12,29,59,60,67,101,102,104]$ and two cognitive DT interventions $[39,85]$. The samples were heterogeneous, since healthy older adults $(n=5)$ and patients $(n=5$; Stroke, Parkinson's disease, Dementia) were included. Results were mixed with regard to positive effects on cognitive, motor or motor-cognitive DT performance. Nonetheless, the authors suggested using multimodality interventions that combine motor and cognitive therapy, to reduce fall risks. Interestingly, also the two reviewed cognitive DT studies $[39,85]$ have been shown to improve DT motor-cognitive performance. To our knowledge, these are the only studies that used a cognitive DT intervention to improve motor-cognitive DT performance. Thus, we did not include them into our review.

Overall, both reviews included different DT studies and healthy as well as patient populations. Thus, it is still unknown under which conditions training might be beneficial for motor-cognitive DT task performance. That is, for example, what kind of DT training, a task specific or rather general motor or cognitive or combined motor-cognitive training might help to improve balance and/or gait and cognitive performance in situations that require managing a cognitive and a standing or walking task simultaneously? Beyond that it remains unclear to what extent the characteristics and complexity of the motor and cognitive task (e.g., visually determined cognitive task [75], standing or walking motor task) might influence DT training success [58].
Up to now, there is no systematic review on training effects of motor-cognitive DT performance for healthy older adults which focus on standing and walking performance considering the effects of different types of training on cognitivemotor DT performance. Thus, we conducted a systematic review to investigate under which conditions older adults benefit from training interventions in motor-cognitive DT situations which require managing a given standing or walking task and a cognitive task. We analysed whether training effects differed with regard to the training programme and/or the task conditions (e.g., standing and walking motor task, complexity/character of secondary cognitive task).

The main questions to be answered were

- Which types of training programmes (specific versus general training, ST versus DT training) are most effective to improve DT performance? That is, which training effects on ST and DT motor performance while standing or walking were reported and which benefits for cognitive performance under ST and DT conditions were described with regard to different types of intervention?

- Do the training effects differ between different complex cognitive tasks/cognitive tasks with different characteristics and between different standing and walking tasks?

The results of this review have an important impact on developing and conducting appropriate DT interventions, especially in fall prevention, that might be used to maintain motor and cognitive performance in complex daily life situations and thus the functional abilities of older adults.

\section{Methods}

\section{Database sources and search terms}

Databases were systematically searched by using OVIDsp to search in Medline (1950 to 2011, Week 52), EMBASE (1980 to 2011, Week 52) and PsycINFO (1806 to 2011, Week 52). All searches were undertaken using the same search strategy and key terms (cf. Table 1 for search stages and the number of paper retained in each database and search stage). The search query consisted of a combination of relevant text words (aligned within Thesaurus). The methodological filters used were: healthy older adults $\geq 65$ years, no brain injuries or cognitive declines, no physical impairments (e.g., using a cane or a walker), no chronic diseases, and English language peer-reviewed articles (cf. Table 1 for more details). In this systematic review a DT training study was defined as a measure of DT performance at pre- and post-test and an intervention that either focused on specific (taskrelated) ST or DT training or on a general (not task 
Table 1 Search stages, number of paper retained in each database, and key terms used in the search

\begin{tabular}{|c|c|c|c|}
\hline \multirow[t]{2}{*}{ Search stage } & \multicolumn{3}{|c|}{ Papers retained } \\
\hline & Medline & EMBASE & PsycINFO \\
\hline $\begin{array}{l}\text { 1. age or old } \$ \text { or "advanced age" or senior } \$ \text { or elder } \$ \text { or geriatric } \$ \\
\text { or aged or eldest or geronic or aging }\end{array}$ & $5,071,761$ & $3,934,136$ & 577,515 \\
\hline $\begin{array}{l}\text { 2. "corresponding task } \$ " \text { or "coupled task } \$ " \text { or "dual task } \$ " \text { or } \\
\text { "dual task paradigm\$" or "secondary task" or "conflicting task" } \\
\text { or "task priori\#ation" or "inattentional blindness" }\end{array}$ & 2,141 & 2,743 & 2,986 \\
\hline $\begin{array}{l}\text { 3. practice or train } \$ \text { or improve } \$ \text { or exercise or treatment or } \\
\text { intervention }\end{array}$ & $3,542,216$ & $4,936,819$ & 793,454 \\
\hline 4. motor or move $\$$ or balance or posture or standing or walking & 643,441 & 726,053 & 179,721 \\
\hline 5. Combination of 1 and 2 and 3 and 4 & 195 & 187 & 75 \\
\hline Assessment based on title and abstract & 55 & 42 & 29 \\
\hline Assessment based on reading the whole paper & 40 & 25 & 9 \\
\hline Total number of included papers & 13 & & \\
\hline
\end{tabular}

related) ST or DT intervention. The following four assessment criteria have been used:

(1) Investigation of at least one motor task (in a DT setting)

(2) Assessment of DT performance (pre-post design)

(3) Conduction of an intervention (physical or combined motor-cognitive training programme)

(4) Investigation of healthy or balance impaired (fallers) older adults in either a randomized control trail (RCT), an experimental-control group design or an old-young comparison

The authors screened the title and the abstract of the selected articles for the inclusion criteria. Full articles were retrieved if they were relevant, or if it was unclear, whether they were relevant after reading the abstract. The included articles were assessed by the authors independently.

\section{Selection criteria}

After pre-screening a total of 46 studies were eligible out of the three data bases (cf. Table 1 for the search strategy). Based on titles, studies in languages other than English were excluded $(n=1 ; 78)$ along with case reports $(n=4 ;[1,6,47,65])$, studies not concerning motor training interventions to improve standing or walking tasks $(n=17 ;[7,8,10,13,18,42-44,46,49,54$, $64,68,71,81,90,91,97])$, not using a pre-post test or agecomparison design, $(n=4$ : $[22,40,89,98])$, not conducted with older adults $(n=1 ;[37])$ or not focusing healthy older adults $(n$ $=6 ;[3,22,24,82,99,100]$ ) were also excluded (cf. Table 5 for excluded studies in the Annex). Finally, a total of 13 studies met all inclusion criteria (cf. Table 2) and were included in the review (last search update on December 31, 2011).

To avoid a reporting bias, we included randomized controlled trails (RCTs) as well as relevant age comparison studies and controlled pre-post designs (CPPD; without a randomization procedure). The classification of the quality of the included studies was done with modified criteria by van Tulder and colleagues [83] for Cochrane reviews (cf. criteria a-i in Table 3). We added three more criteria which were not included by van Tulder and colleagues [83] but relevant for the outcomes of this review: $\mathrm{j}$, appropriate description of the intervention (content, intensity, duration); $j$, appropriate description of the measurements; 1 , adequate report of measurement results and statistics. For each criteria $(n=12)$ one point was given and points were summarized to the quality score. Eight out of the points were given for the modified van Tulder et al. [83] criteria and three for the additional methodological criteria. In sum the highest quality would be reached by $8+3$ point. The quality score needs to be $\geq 5+2$ for a study of high quality (and $\geq 4+2$ for the age comparison studies, because some of the methodological criteria like randomization are not relevant for agecomparison studies). All studies with a score of $<5+2$ are defined as of lower quality. Moreover we reported some general methodological issues (cf. column general marks).

Six of the included studies were RTCs $[23,29,66,67,80$, 93]. All six studies, except that of Hall et al. [23], were of high quality. Also the two identified age comparison studies conducted by Dault and Frank and Doumas and colleagues $[18,19]$ reached high-quality scores. From the five included CPPD studies [9, 28, 35, 79, 104], only one [104] could be considered high quality. The other four $[9,28,35,79]$ had methodological deficits as described in Table 3.

\section{Results}

Classification of included studies

We classified included studies in an overview table (cf. Table 2). Data of interest were:

- Sampling (age comparison [AC], RCT, CPPD) 


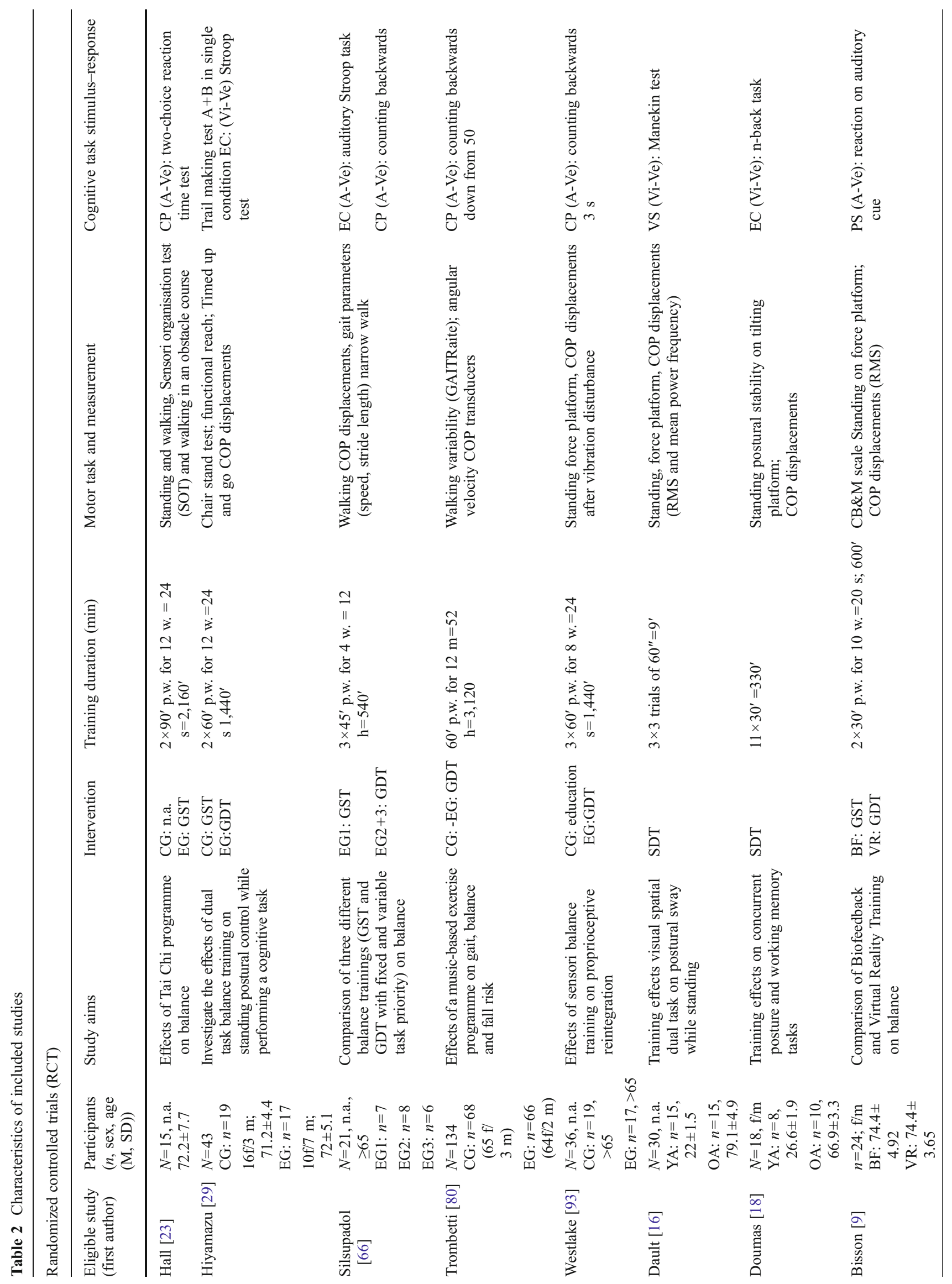




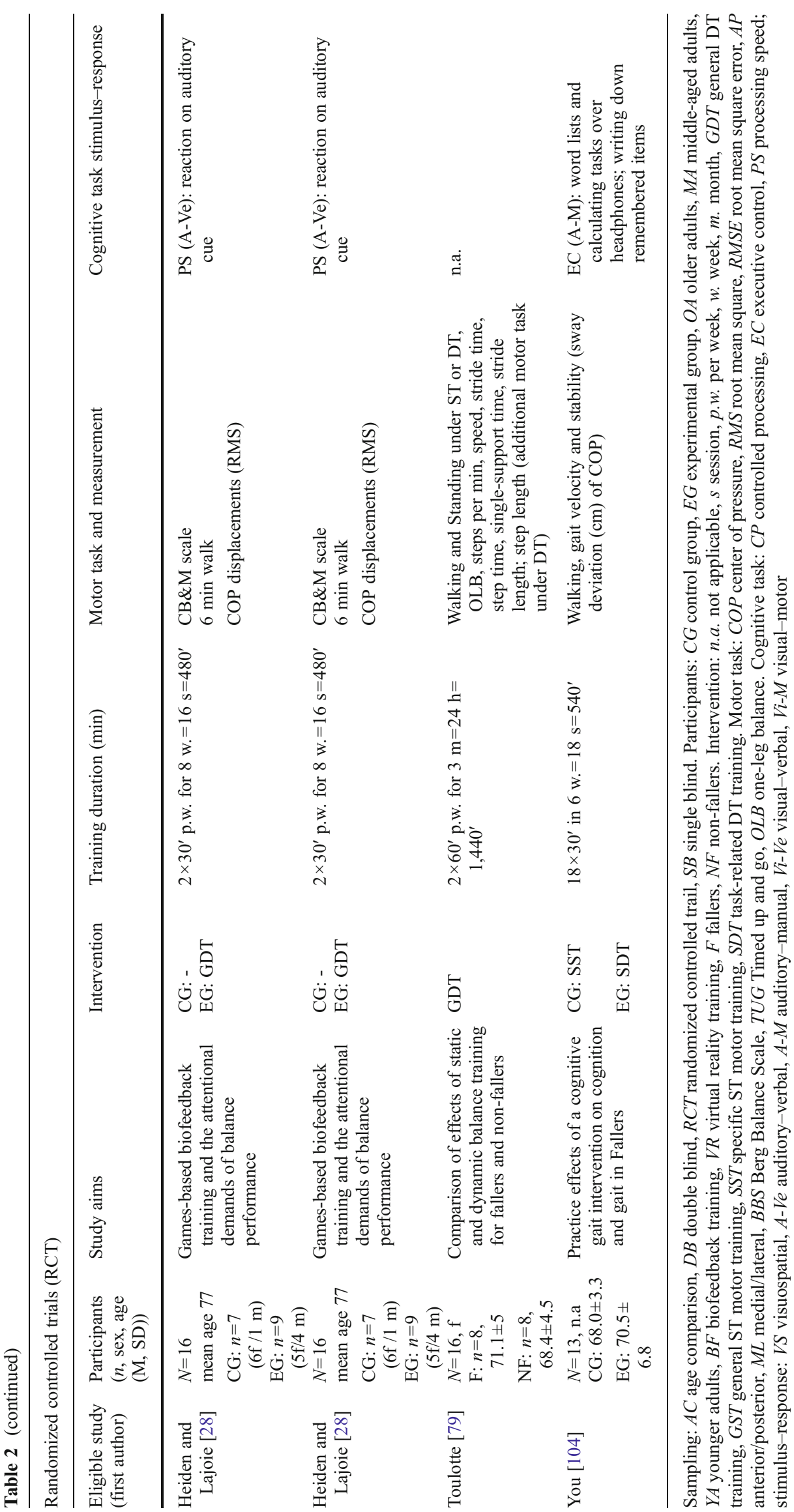


- Participants (number of participants, age, gender)

- Study aim

- Type of intervention and training duration

- Motor task (standing or walking) and measurement

- Cognitive task (executive control, controlled processing, processing speed, visuospatial task; cf. [14]) measurement

- Type of stimulus and stimulus-response in the cognitive task (auditory, verbal, visual, motor)

The differentiation of the cognitive task was based on the categorization suggested by Colcombe and Kramer [14] who differentiated between processing speed task (i.e., tasks that represent a measure of low-level neurological functioning such as simple reaction time), visuospatial processing tasks (i.e., tasks that measure the ability to transform or remember visual-spatial information), controlled processing tasks (i.e., tasks that require at least some cognitive control), and executive control tasks (i.e., tasks related to planning, inhibition and scheduling of mental processes).

The included studies (cf. Table 2) were heterogeneous and differed in:

- Methodological quality (cf. Table 3).

- Sample size (from 13 [104] up to 134 [80] participants).

- Investigated motor tasks: Seven studies investigated standing tasks [9, 16, 17, 28, 29, 35, 93]. Four studies examined walking [66, 67, 80, 104], and two studies combined walking and standing tasks $[23,79]$ to analyse intervention effects on DT performance.

- Measurements of motor performance: A variety of different tests and clinical assessments were used to describe motor performance while standing and walking. To examine motor performance in standing tasks, four studies [28, 29, 35, 79] used clinical tests, like the Berg Balance Scale (BBS), Timed up and go (TUG) or the Community Balance and mobility scale (CB\&M). Seven studies investigated standing motor performance by the use of biomechanical assessments like force platforms or other measurements of COP sway displacements $[9,16,17,23,28,80$, 93] and two studies [29, 35] used both types of testing. To observe walking performance, three studies used clinical assessments (e.g., TUG [23, 28, 104]) and five studies used measurements to gain precise biomechanical markers like gait variability, velocity, cadende, and stride length - for example, a gait mat with sensors or a kinematic system [66, $67,79,80,104]$. One study [23] used both kinds of testing to report walking performance.

- Complexity of the cognitive task: The majority of studies applied cognitive tasks focusing on controlled processes $(n=5 ;[23,66,67,80,93])$ or executive control tasks $(n=4 ;[18,29,66,104])$. Three studies examined processing speed tasks [9, 28, 35] and only one study used a visuospatial task [16]. Another study did not specify the cognitive task [79].

- Stimulus-response of the cognitive task: a preference for verbal responses can be observed with most studies applying an auditory $(n=8 ;[9,23,28,35,66,67,80$, 93]) or a visual cognitive stimulus $(n=3$; $[16,17,23])$ with a verbal response. One study used an auditory task with a motor response [104]. Again one study did not specify the stimulus-response [79].

- Training duration (from nine trials with a duration of 9 min within one session [16] up to about 52 sessions across 12 months with a total duration of 3,120 $\mathrm{min}$ [80].

Description of the types of training interventions used in the included studies

A special focus of this review lain in the analysis of the training interventions used to improve motor-cognitive DT performance and to define a training methodology with greatest effectiveness for motor-cognitive performance under DT conditions. For the type of intervention we differentiated between task-related (specific) training, i.e., either only the examined motor or cognitive task alone (specific ST training [SST]) has been trained or both examined motor and cognitive tasks have been trained simultaneously (specific DT training) in a fixed modality, and general training, i.e., variable task conditions in different modalities have been conducted, such as a general balance training or fall prevention programme or a motor biofeedback training focusing on the motor task (general ST training [GST]), or a general balance/gait training combined with secondary tasks (general DT training [GDT]). Consequently, four types of interventions were identified: (1) GST, (2) SST, (3) GDT, and (4) task-related (specific) DT training (SDT) (cf. see below for a more detailed description).

\section{General ST training}

GST was defined as an intervention in which tasks differed from the ones used in pre- and post-tests, but only one component of the DT situation (here motor performance) was trained. In six studies GST training was used [9, 23, 29, $35,66,67]$. Two studies investigated the effects of GST (general balance or a walking training) on DT walking performance [66, 67], one study focused on the effects of GST on walking and standing performance ([23]; results will be reported for standing and walking), and three studies used GST training to examine specific effects on DT balance performance while standing [9, 29, 35]. 
Table 3 Quality scores and remarks of the included studies

\begin{tabular}{|c|c|c|c|c|c|c|c|c|c|c|c|c|c|c|}
\hline \multirow[t]{2}{*}{ Study } & \multicolumn{12}{|c|}{ Quality criteria } & \multirow{2}{*}{$\begin{array}{l}\text { Quality } \\
\text { score }\end{array}$} & \multirow[t]{2}{*}{ General remarks } \\
\hline & $\mathrm{a}$ & $\mathrm{b}$ & $\mathrm{c}$ & $\mathrm{d}$ & $\mathrm{e}$ & $f$ & $\mathrm{~g}$ & $\mathrm{~h}$ & $\mathrm{i} j$ & & $\mathrm{k}$ & 1 & & \\
\hline \multicolumn{15}{|l|}{ RCTs } \\
\hline Hall b [23] & $\mathrm{x}$ & - & - & - & $\mathrm{u}$ & $\mathrm{u}$ & $\mathrm{u}$ & $\mathrm{u}$ & -2 & $\mathrm{x}$ & $\mathrm{x}$ & (x) & $1+2$ & $\begin{array}{l}\text { k: statistical information missing, } \\
\text { e.g., ES }\end{array}$ \\
\hline $\begin{array}{l}\text { Hiyamazu } \\
\text { [29] }\end{array}$ & $\mathrm{x}$ & $\mathrm{x}$ & $\mathrm{x}$ & $\mathrm{x}$ & $\mathrm{u}$ & $\mathrm{u}$ & $\mathrm{x}$ & $\mathrm{u}$ & - r & $\mathrm{x}$ & $\mathrm{x}$ & $\mathrm{x}$ & $5+3$ & \\
\hline $\begin{array}{l}\text { Silsudapol } \\
\text { [66] }\end{array}$ & $\mathrm{x}$ & $\mathrm{x}$ & $\mathrm{x}$ & $\mathrm{x}$ & $\mathrm{x}$ & $\mathrm{x}$ & $\mathrm{x}$ & $\mathrm{x}$ & $\mathrm{x}$ & $\mathrm{x}$ & $\mathrm{x}$ & (x) & $9+2$ & Small SS \\
\hline $\begin{array}{l}\text { Silsudapol } \\
\text { [67] }\end{array}$ & $\mathrm{x}$ & $\mathrm{x}$ & $\mathrm{x}$ & $\mathrm{x}$ & $\mathrm{x}$ & $\mathrm{x}$ & $\mathrm{x}$ & $\mathrm{x}$ & $\mathrm{x}$ & $\mathrm{x}$ & $\mathrm{x}$ & (x) & $9+2$ & Small SS \\
\hline $\begin{array}{l}\text { Trombetti } \\
{[80]}\end{array}$ & (x) & $\mathrm{u}$ & $\mathrm{x}$ & $\mathrm{x}$ & $\mathrm{u}$ & $\mathrm{u}$ & $\mathrm{x}$ & $\mathrm{x}$ & $\mathrm{x}$ & $\mathrm{x}$ & $\mathrm{x}$ & $\mathrm{x}$ & $5+3$ & Randomization process unclear \\
\hline $\begin{array}{l}\text { Westlake } \\
\text { [93] }\end{array}$ & $\mathrm{x}$ & $\mathrm{x}$ & - & $\mathrm{u}$ & $\mathrm{x}$ & $\mathrm{x}$ & $\mathrm{x}$ & $\mathrm{x}$ & - : & $\mathrm{x}$ & $\mathrm{x}$ & (x) & $6+2$ & \\
\hline \multicolumn{15}{|c|}{ Age comparison designs } \\
\hline Dault [16] & & & & & $\mathrm{x}$ & $\mathrm{x}$ & $\mathrm{x}$ & $\mathrm{x}$ & - r & $\mathrm{x}$ & $\mathrm{x}$ & $(\mathrm{x})$ & $4+2$ & 6 trails only $-\mathrm{f}+\mathrm{g}$ implicit \\
\hline $\begin{array}{c}\text { Doumas } \\
{[18]}\end{array}$ & & & & & $\mathrm{x}$ & $\mathrm{u}$ & $\mathrm{x}$ & $\mathrm{x}$ & - & $\mathrm{x}$ & $\mathrm{x}$ & $\mathrm{x}$ & $4+2$ & $g$ suggested out of $d$ and $f$ \\
\hline Bisson [9] & - & $\mathrm{u}$ & $\mathrm{x}$ & $\mathrm{u}$ & $\mathrm{x}$ & $\mathrm{u}$ & $\mathrm{x}$ & $\mathrm{x}$ & - r & $\mathrm{x}$ & $\mathrm{x}$ & (x) & $4+2$ & $\mathrm{~g}$ suggested out of $\mathrm{d}$ and $\mathrm{f}$ \\
\hline $\begin{array}{l}\text { Heiden and } \\
\text { Lajoie [28] }\end{array}$ & $\mathrm{x}$ & - & - & $\mathrm{x}$ & $\mathrm{u}$ & $\mathrm{u}$ & $\mathrm{u}$ & $\mathrm{x}$ & - r & $\mathrm{x}$ & $\mathrm{x}$ & (x) & $3+2$ & Randomization process was weak \\
\hline Lajoie [79] & - & - & $\mathrm{x}$ & $\mathrm{u}$ & $\mathrm{u}$ & $\mathrm{u}$ & $\mathrm{u}$ & $\mathrm{x}$ & - r & $\mathrm{x}$ & $\mathrm{x}$ & $\mathrm{x}$ & $2+3$ & \\
\hline Toulotte [79] & & & $\mathrm{x}$ & $\mathrm{u}$ & $\mathrm{u}$ & $\mathrm{u}$ & & $\mathrm{x}$ & - & (x) & $\mathrm{x}$ & (x) & $2+1$ & $\begin{array}{l}\text { a: classification in faller/non-faller; k: } \\
\text { statistical information missing, e.g., ES }\end{array}$ \\
\hline You [104] & $\mathrm{x}$ & $\mathrm{u}$ & $\mathrm{x}$ & $\mathrm{u}$ & $\mathrm{x}$ & $\mathrm{x}$ & $\mathrm{x}$ & $\mathrm{x}$ & - & $\mathrm{x}$ & $\mathrm{x}$ & (x) & $6+2$ & \\
\hline
\end{tabular}

$x$ "yes" score, $u$ "unclear" score, free fields "not relevant"; - "no" score, $(x)$ "was done, but with general remarks", RCT ES effect size, $S S$ sample size

a - acceptable method of randomization; $\mathrm{b}$ - concealed treatment allocation; c - similar group values at baseline; $\mathrm{d}$ blinded assessor; e - avoided or similar cointerventions; $\mathrm{f}-\mathrm{ac}-$ ceptable compliance; $g$ - acceptable dropout rate; $\mathrm{h}$ - similar timing of the outcome assessment in all groups; i - intention-totreat analysis; $\mathrm{h}$ - appropriate description of the intervention (content, intensity, duration); $\mathrm{j}$ appropriate description of the measurements; $\mathrm{k}$ - adequate report of measurement results and statistics
Types of GST different between the studies: Two studies used a biofeedback training $[9,35]$. In the biofeedback training of Lajoie [35] (60 min, two times/week, 8 weeks), participants were able to view their own COP displacements by the movement of a cursor and were asked to minimize the cursor movements. The feedback was reduced from $100 \%$ to $30 \%$ from the first to the last session. Bisson and colleagues [9] compared a similar biofeedback training protocol (GST) with a virtual reality training (GDT, for the virtual reality training cf. GDT) (30 min, two times/week, 10 weeks). Participants were instructed to move a cursor to specific directions viewed on a screen by controlling their COP displacements. One study [23] used a general Tai Chi intervention $(90 \mathrm{~min}$, two times/week, 12 weeks) compared to a not further described control group. In three studies [29, 66, 67] GST (general balance exercises combined with walking elements [66, 67] or strength training [29] served as a control condition to compare GST training with GDT training (e.g., calculating or visual observing tasks; cf. below) gains ([29]: 24 sessions, $60 \mathrm{~min} / \mathrm{session}[66,67]: 12$ sessions, $45 \mathrm{~min} / \mathrm{session})$. The goal of these three interventions was to investigate whether GDT training $([66,67]$ : DT balance training either with fixed or variable priority; [29]: balance and strength training plus calculating or visual observing tasks) was more effective to improve DT balance performance for standing [29] and walking $[66,67]$ tasks than GST training.

\section{Specific ST training}

SST includes the training of the motor task that is also used as outcome measure. Only one study [104] investigated the effect of an SST to investigate DT walking performance, and no SST study for standing was found. Here, the SST served as a control group compared to SDT training. The intervention consisted of walking while listening to music over headphones as a placebo intervention (SST) and of walking while performing an additional cognitive task presented via headphones (SDT, cf. below) (18 sessions, $30 \mathrm{~min} / \mathrm{session}$ ).

\section{General DT training}

GDT means that the training includes a variety of DTs in combination with balance or walking performance. The training tasks differ from the test situations. These kinds of interventions were identified in eight of the included studies $[9,28,29,66,67,79,80,93]$. Four studies investigated the influence of GDT on DT balance performance during standing conditions [9, 28, 29, 93]. One study examined both, effects on standing and walking performance ([79] results will be reported for standing and walking), and three studies examined effects of their GDT on DT walking performance $[66,67,80]$. 
Again, as mentioned for GST, intervention programmes differed between the studies.

Most of the interventions $(n=6[29,66,67,79,80$, 93]) performed a general balance or fall prevention programme including balance, strength and walking tasks combined with different cognitive tasks. Two of them used tasks like reciting poems, calculating numbers, or visual spatial cognitive tasks ([93]; 24 sessions, $60 \mathrm{~min} /$ week, 8 weeks; [29]; 24 sessions, $60 \mathrm{~min} / \mathrm{session}$ ], another two conducted a task priority training for fall prevention ([66, 67]: 12 sessions within 4 weeks, $45 \mathrm{~min} / \mathrm{session}$ ) related to Bherer and colleagues [8], that is, either performing DTs with a fixed priority or with switching priority between the tasks (variable priority). As mentioned above, these task priority training groups were compared with a GST training group. One study [80] combined different balancing and walking task together with cognitive music tasks, e.g., react on rhythmic changes (52 sessions of $60 \mathrm{~min}$ ). One study did not specify the content of the additional cognitive tasks in the fall prevention intervention ([79]; two sessions of $60 \mathrm{~min} /$ week, 12 weeks).

Finally, two studies $[9,28]$ used virtual reality games as a DT biofeedback training. Bisson and colleagues [9] asked their participants to juggle a virtual ball with additional real-time visual feedback (two sessions of $60 \mathrm{~min} /$ week, 8 weeks) and [28] used a virtual tennis game (two sessions of $30 \mathrm{~min} /$ week, 8 weeks). In both studies, participants were asked to compensate COP displacements simultaneously, i.e., while observing a virtual ball and using arm movements to hit the ball.

\section{Specific Dual Task training (SDT)}

Three studies used SDT to examine its influence on $D T$ balance performance while standing $[16,17]$ or walking [104]. As described for the SST, we categorized training as specific when the training tasks are the same as the tested tasks. In all these studies, participants trained first under ST and then under DT conditions, so that the tasks increased in difficulty or complexity over the course of training. One study used a control group design [104] and two an age comparison design [16, 17]. Both age comparison studies combined standing tasks ([17]; ST and DT standing on a fixed or moving platform; [16]; standing on a force platform) with secondary cognitive tasks. The secondary cognitive tasks during the training interventions differed between the studies. Dault and Frank [16] used a visuospatial task (Manekin Test; 9 min with nine 60-s trials), Doumas and colleagues [17] an n-back memory task (11 sessions of $30 \mathrm{~min}$ each), and You and colleagues [104] a memorizing computing task (18 sessions of $30 \mathrm{~min}$ over 6 weeks).
Results sorted by training types

In the following, results will be reported separately for standing and walking outcome tasks with regard to the four different training interventions. Standing and walking were separated because of the different complexity of motor control [25] and movement coordination [25] that are needed to maintain static (standing) or dynamic (walking) balance (Table 4).

Effects of GST training on motor and cognitive performance under single-task (ST) and DT conditions

Standing Effects on DT and ST standing performance

All four GST balance training interventions which examined DT standing performance $[9,23,29,35]$ failed to improve DT balance performance while standing (with a Tai Chi intervention [23], with a balance programme [29], and with an ST biofeedback training $[9,35])$. Three studies, however, found improvements of motor performance under ST condition [9, 23, 35], whereas one study [29] found no effects on ST motor performance.

Effects on DT and ST cognitive performance

Effects on cognitive performance under ST and DT conditions were, unfortunately, not systematically reported or investigated. Only the two GST studies that used biofeedback reported increased performance in a cognitive processing speed task (auditory-verbal task) [9, 35] under DT conditions. Cognitive ST effects were only investigated by Hiyamazu et al. [29] (trail making a and b test) showing no effect of GST.

\section{Walking Effects on DT and ST motor performance}

In contrast to the results for standing performance, two out of three $[23,66,67]$ studies that investigated the effects of GST on ST and DT motor performance while walking found positive effects on DT motor performance. That is, two studies $[66,67]$ found improvements in walking performance in a narrow walk while counting backwards in $3 \mathrm{~s}$ (auditory-verbal controlled processing task) after their general balance and coordination programmes. GST by Hall and colleagues [23], however, failed to improve walking performance in an obstacle course under DT conditions (verbal reaction on a green light; visual verbal controlled processing task) after a Tai Chi training, whereas the nonexercising control group (no information given) increased DT walking performance. Also, effects on ST walking performance were found by the two studies of Silsupadol and colleagues [66, 67] and by Hall and co-workers [23] for the control (without an intervention) and experimental group.

Effects on ST and DT cognitive performance

All three GST interventions which investigated training impacts on gait $[23,66,67]$ did not report any positive 


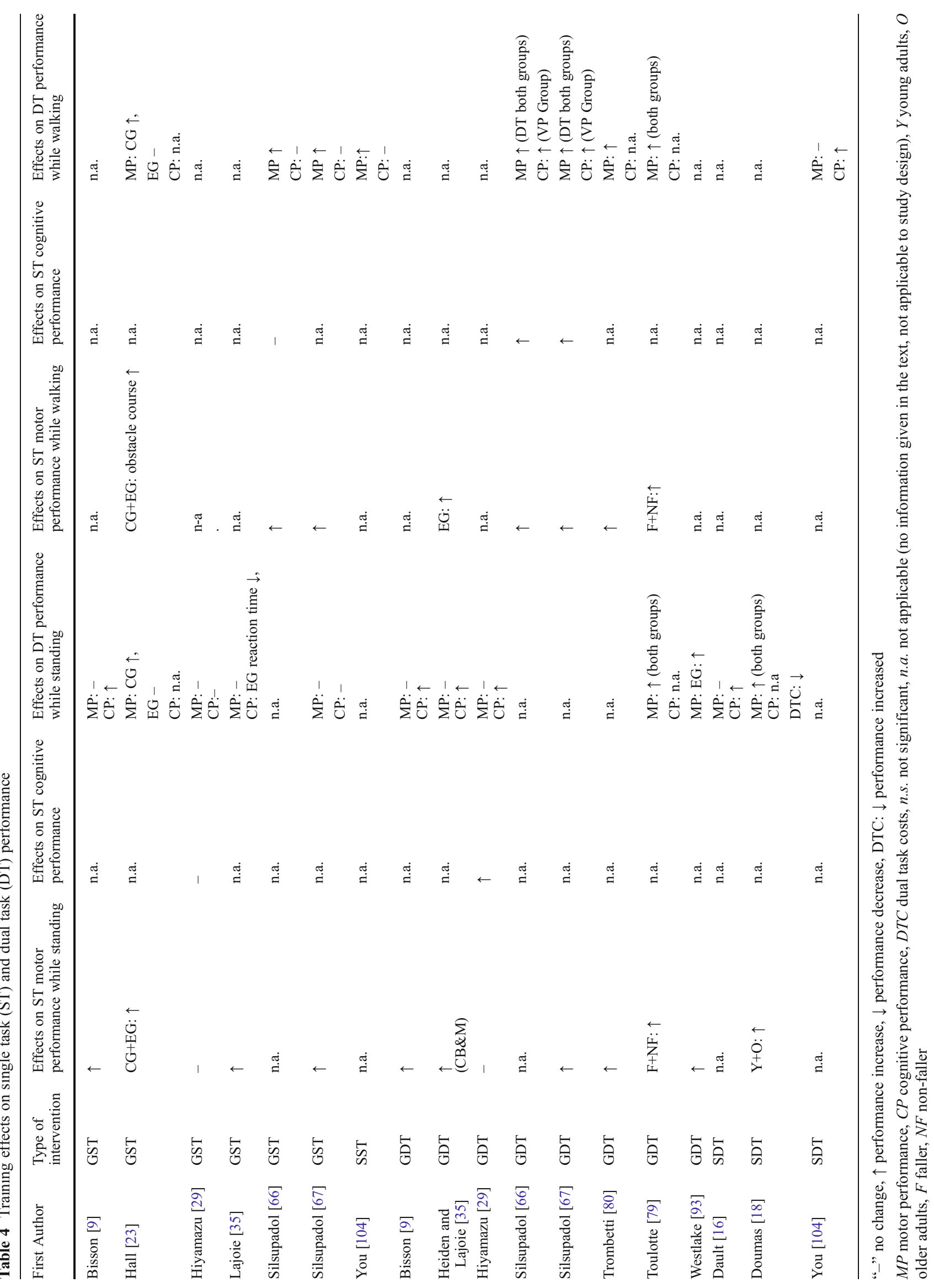


outcomes for DT cognitive performance for auditory verbal controlled processes tasks while walking. Effects on ST cognitive performances (auditory-verbal calculating controlled processing task) were only evaluated by Silsupadol and colleagues (a) [66] failing to show positive effects.

\section{Effects of SST training on motor and cognitive performance under ST and DT conditions}

Standing No study available.

Walking Effects on DT and ST motor performance

The only SST study [104] examined training effects on walking performance showing improved DT walking performance (walking variability) after training.

Effects on DT and ST cognitive performance

The cognitive performance (remembering auditory presented word lists or calculating tasks) under DT conditions did not improve in the study of You et al. [104] ST motor or cognitive performance was not reported.

\section{Effects of GDT training on motor and cognitive} performance under ST and DT conditions while standing

Standing Effects on DT and ST motor performance

Only two $[79,93]$ out of five studies $[9,28,29,79$, 93] found or reported improvements of motor performance while standing under DT conditions after balance and coordination exercises with nonspecific DTs like reciting poems or calculation tasks. Toulotte and colleagues [79] showed improved one leg balance for their examined fallers and non-fallers (DT condition was not further described). In the study of Westlake and Culham [93], the participants were able to compensate COP displacements while performing a visual-verbal letter counting task (controlled processing). Under ST conditions all GDT interventions, except of Hiyamazu and co-workers [29], were able to improve standing performance.

Effects on DT and ST cognitive performance

Improvements of DT cognitive performance while performing a standing task were reported by three of the five studies $[9,28,29]$. They all used force platform measurements to report COP displacements or COP sway. Bisson et al. [9] and Hiyamazu et al. [29] used an auditory-verbal processing speed task, whereas [29] used a visual-verbal executive control task (Stroop task) . Only Hiyamazu and colleagues [29] investigated cognitive performance under ST conditions. Their intervention group improved in a trail making test.
Walking Effects on DT and ST motor performance

All four studies [66, 67, 79, 80] stated improvements in the examined gait tasks (i.e., cadence, stride length, single support time, walking speed, narrow walk,) after a GDT intervention. Also, under ST conditions, all four GDT interventions $[66,67,79,80]$ improved walking performance (e.g., walking speed, narrow walk).

Effects on DT and ST cognitive performance

Only two studies $[66,67]$ described effects of GDT on cognitive performance under DT conditions. Silsupadol and co-workers [66, 67], who trained a fixed focus of attention in comparison to a variable focus of attention, reported improvements in a task requiring controlled processing. In addition, Silsupadol et al. [66] used a transfer task (executive control task [Stroop task]), but failed to show transfer effects. Under ST conditions positive results for cognitive performance (auditory-verbal counting backwards task; controlled processing) were reported by only the study of Silsupadol et al. [66].

Effects of SDT training on motor and cognitive performance under ST and DT conditions

Standing Effects on DT and ST motor performance

Whereas Doumas and colleagues [18] reported improvements for older and younger adults to compensate COP displacements while performing an executive visual-verbal n-back task (executive control) after a SDT training, Dault and Frank [16] did not find increased motor performance (COP displacements) while performing a visual-verbal visuospatial task. In addition, only Doumas and colleagues [18] investigated ST performance while standing. They found improvements for older as well as for younger adults under ST condition on a fixed or moving platform (reduced COP displacements).

Effects on DT and ST cognitive performance

Although not able to show positive effects of SDT on motor performance under DT conditions, the intervention of Dault and Frank [16] led to positive effects on cognitive performance (visual-verbal visuospatial task). Doumas and colleagues [18] did not report any results for cognitive performance; however, they found decreased DTC (executive n-back task) in their test situation for the older and the younger participants. Both SDT studies did not report ST cognitive performance while standing.

\section{Walking Effects on DT and ST motor performance}

As reported above, You and co-workers [104] used a SDT in comparison to a SST. They trained the examined cognitive task (remembering auditory presented word lists or calculating tasks) while walking and did not find positive effect for DT walking performance in the experimental group (but in the control group). 
Effects on DT and ST cognitive performance

You et al. [104], however, confirmed positive improvements for the cognitive task (auditory-manual executive task) under DT conditions. Results for motor or cognitive performance under ST conditions were not reported.

Summary of the main results of the training interventions and study aims

The main questions were as follows: which types of training programmes (specific versus general training, ST versus DT training) are most effective to improve DT performance and which training effects on ST and DT motor performance while standing or walking were reported?

Benefits on motor performance under ST and DT conditions:

- DT motor performance while standing was improved by three of the eight included training interventions. Two of them used GDT [79, 93] and one SDT [18]. The GDT improved one leg balance in fallers and non-fallers [79] and increased postural stability after mechanical induced displacements of the COP [93]. The SDT improved postural sway on a moving force platform [18].

- DT motor performance while walking was improved by seven of the 13 interventions, either by the use of ST $(n=2$ GST $[66,67] ; n=1$ SST [104] or DT $(n=4$ GDT $[66,67,79,80])$ interventions. The only type of intervention that revealed no effect on DT walking performance was SDT ([104]; note that only one study is available). The main motor outcomes were improved walking parameters like cadence for GDT [79], gait variability and walking speed (GDT [66, 67, 79, 80], GST [66, 67], SST [104], and narrow walk GST and GDT $[66,67])$.

- Additional benefits on ST motor performance while standing were reached by all interventions except of the GST and GDT by Hiyamazu and colleagues [29] and the SDT by Dault and Frank [16].

- Positive changes on ST walking performance were reached by all of the GST [23, 66, 67] and GDT [28, $66,67,79,80]$ studies which included ST walking measurements. Only for the SST and SDT training of You et al. [104] no changes in ST walking performance were reported.

Benefits on cognitive performance under ST and DT conditions:

- DT cognitive performance while standing was improved by five out of six studies using GST [9, 35], GDT [9, 28, 29] or SDT [16]. Positive effects were found for processing speed of auditory-verbal tasks [9, 28], a visualverbal executive Stroop test [29] and a visual-verbal visuospatial task [16]. The SDT of Doumas and colleagues [18] did not report DT cognitive performance separately, but they found decreased DTC for an executive n-back task. Effects of SST on DT cognitive performance were not examined.

- Eight studies examined DT cognitive performance while walking. Most of the ST training studies (three studies using GST [23, 66, 67] and the study performing SST [104]) found no benefits on cognitive DT abilities. In contrast, three of the eight GDT programmes [28, 66, 67] and the SDT conducted by You et al. [104] increased the cognitive DT performance, either in an auditoryverbal processing speed task [28] or in more complex controlled processing tasks [66, 67, 104].

- ST cognitive performance while standing was only examined in two studies by the use of different types of interventions: Hiyamazu et al. [29] for GST and GDT (trail making test), Doumas et al. [18] for SDT (n-back executive control task). Again, effects of SST on DT cognitive performance were not examined.

- Only one walking study [66] reported improved ST cognitive performance after GDT. No SST or SDT investigation analysed or reported ST cognitive performance measurements. For GST only one study [29] examined ST cognitive performance and failed to induce positive effects.

- In sum, intervention effects on cognitive performance were less investigated (only in nine studies for DT conditions $[9,16,18,28,29,35,66,67,104]$ and in two studies for ST conditions [29, 66]) than on motor performance. Based on the results, one might assume that there is an advantage in DT training, either GDT or SDT, to improve cognitive performance. Due to the low evidence, results have to be interpreted carefully. In addition, most studies used controlled processing tasks with an auditory-verbal stimulus-response. Thus, it is difficult to state a clear advantage or benefits of a specific task complexity or stimulusresponse condition.

\section{Discussion}

The aim of this review was to investigate whether healthy older adults benefit from training interventions in DT situations with the need of balance control while standing or walking. We focused on the training effects with regard to the type of training (specific or general ST or DT training) and the task conditions (standing or walking) as well as the complexity of the secondary cognitive task. The results of the different training interventions were heterogeneous and differed with regard to the training protocol (general or specific ST/DT training) and between standing and walking. 
First of all, most studies used a GDT $(n=8)$, followed by a GST $(n=6)$, a specific DT training $(n=3)$ and an SST $(n=1)$. Whereas all four types of training interventions were used in walking studies, no SST has been conducted for standing.

\section{Effects on motor performance}

When looking at general and specific ST training, it becomes clear that ST motor training seems not to be beneficial to improve standing performance under DT conditions (note: no SST for standing is available). Because two GST studies [28, 29] used clinical assessments, where standing was part of a comprehensive test battery, and because Hiyamazu and colleagues [29] did not find any improvements, one can only suggest that GST improves functional capacities while standing. One has, however, to consider that there were no STT, but five GST studies for standing available.

On the contrary, ST training seems to be (at least partly) able to benefit walking performance, either GST (two out of three studies [66, 67]: walking in a narrow walk) or SST [104]. Surprisingly, the not further described control group in the study of Hall and colleagues [23] improved DT performance in an obstacle avoidance course. This might be due to the design of the training programme or due to the outcome measures used to asses DT performance.

We can only speculate why ST training is more beneficial to improve walking than standing performance under DT conditions. GST studies, however, were able to improve ST standing (four out of five studies) and walking (three out of three studies) performance (note: no results reported for specific ST standing interventions). This was true for ST biofeedback [9, 35] and Tai Chi [23] training in standing studies and for balance and coordination trainings in walking studies $[66,67,104]$. This might hint to the fact that a motor training (performed as a GST) particularly improves the trained tasks, i.e., motor performance under ST conditions. Whether this is also true for SST cannot be answered by this review (no studies available), might be, however, plausible to be assumed.

Moreover, specific DT training studies are rare (only three studies used this type of intervention $[10,16,18])$. Effects of specific DT training on motor performance under DT conditions are again heterogeneous. Only one study [18] reported positive effects on balance performance while standing under ST and DT conditions. The positive results of Doumas et al. [18] might point to the importance of the exercise load, as they systematically increased the task difficulties across the 11 training sessions. As shown by previous studies $[13,18,36]$ increasing task difficulties or progressive demands of motor performance seem to be important factors to gain improvements in physical or cognitive functions. Therefore, an appropriate stimulus which is modulated with rising demands might gain higher training effects than training with the same cognitive load or motor demands. The only walking study using a specific DT training [104] gained no effects on walking performance.

The most promising approach to reach motor benefits under DT and ST conditions seem to be a GDT programme. Here, two $[79,93]$ out of six standing and all four $[66,67$, $79,80]$ walking studies reported positive effects on standing or walking performance under DT conditions. These results are in line with previous findings of the review by Pichierri et al. [56]. Again, our results indicate that GDT is more effective to improve walking than standing performance. In remains unclear why the programmes show more benefits on walking conditions than on standing performance. Walking is a more complex task than standing. Therefore it might be easier to improve standing tasks. One might assume that standing is not a challenging task and does not need as many resources as walking. Following this hypothesis, the standing performance of healthy older adults might be (already) on a high level and in conclusion cannot be further improved. On the other hand, one has to consider that the recent literature discussed a lot of issues with balance performance measurements while standing (e.g., reliability of COP sway measures and validity for older adults $[15,34,77]$, whereas the gait analysing systems seem to be able to gain more qualitative data sets i.e., for cadence, stride length, single support time and walking speed (e.g., [84]). Future DT research using standing tasks should validate performance measures under DT conditions and proof whether they are reliable for the investigated cohort.

A further explanation for the positive outcomes of the walking studies might be that in two of them $[66,67]$ the attentional focus has to be continuously switched (variable task priority). In line with Bherer and colleagues [7, 8], Silsupadol et al. $[66,67]$ have shown that DT training with variable task priorities on both tasks seems to be more effective than self-selected or fixed task priority training. It resulted in positive lasting effects on DT motor and cognitive performance [66]. Also, cognitive DT studies [8, 39] that investigated training with different task priorities have shown that training with a variable focus on tasks was successful to increase divided attention performance and reduce DT costs. However, it has to be noted that some of the studies which were included here, like those conducted by Silsupadol et al. [66, 67], used small sample sizes, and therefore additional future research of the effects of this kind of intervention protocol is needed.

A GDT showed also high benefits on motor performance under ST conditions in five out of seven standing studies programmes $[9,28,79,80,93]$ and in all four walking studies $[66,67,79,80]$. The training of balance performance under DT conditions is more complex for motor control and 
coordination than under ST conditions. DT training requires more motor and cognitive resources than ST training, and in turn, it probably trains attentional control, resource allocation and/or task automation. the summary of the results of DT training programmes gives evidence that some training programmes (GDT and SDT) which showed the most benefits on motor and cognitive DT performance) follow the principle of specificity [45] The Specificity Principle states that training must be conducted from highly general training to highly specific training. It also implies that improvements in a particular exercise or skill are task specific and a result of training or performing that specific exercise or skill. To be a good cyclist, cycling has to be trained, to reveal good specific motor-cognitive DT performance the training has to be either task specific and has to be trained at least under DT conditions. In line with this theory all specific training interventions (SDT) and most of the GDT which were used to improve DT performance resulted in positive effects.

Age is assumed to be associated with reduced processing efficiency (e.g., nerve conduction speed, fluid intelligence) [51] and sensory and motor aspects of performance are increasingly in need of cognitive control and supervision with advancing age [38]. Thus, a given task likely exerts a higher demand on the attentional resources of older as compared to younger adults to achieve the same performance level. A better DT or ST motor performance after training can therefore be interpreted as less attention needed to perform one or two of the trained tasks. Motor practice might lead to automation of the task followed by a release of cognitive resources. Therefore, training interventions might be most beneficial if they improve motor performance to a degree that the motor task is performed more automated and frees up mental resources. We suggest that the high impact on different systems organizing motor control and cognition during GDT interventions leads to greater benefits on resource allocation than a task specific or ST training which only influences one modality. Following this argumentation, general DT practice might free up cognitive resources that were previously used to monitor motor and/or cognitive performance and will also improve performance under ST conditions.

However, it is still unclear which dose-response relationships are most effective to improve DT motor-cognitive abilities in older adults. The training duration is one factor that might influence the results. One might assume that lasting effects of motor adaptions require a certain duration of the intervention. The programmes which were described in this review differed in their duration from nine trials with a duration of 9 min within one session [16] up to about 52 sessions across 12 months with a total duration of $3,120 \mathrm{~min}$ [80]. When summarizing the results with regard to the training duration, it becomes obvious that the programmes which improved DT standing abilities lasted between 11 sessions with $330 \mathrm{~min}$ [18] and 24 sessions with 1,440 min [93]. Programmes that improved walking DT performance lasted from 12 sessions with $540 \min [66,67]$ up to 52 sessions [80]. Interestingly, positive effects on motor and cognitive performance under DT conditions were already gained with relatively short training protocols (330 $\min [18] ; 540 \min [66,67])$. This is a further hint that besides the duration, the content for the training protocol (specific/general ST/DT training) might be an important, or even a more important, aspect to induce benefits on DT performance.

\section{Effects on cognitive performance}

Unfortunately, many studies did not report effects on DT cognitive procedures. ST training (general or specific) seems to have only weak effects on cognitive performance under ST or DT conditions. Only GST biofeedback training $[9,35]$ was shown to improve processing speed under DT conditions while standing with an auditory verbal task. Positive effects on ST cognitive performance were reported in one standing ([29], trail making test) and one walking study ([66]: auditory verbal controlled processes). For specific DT training, increased cognitive performance under DT was found in both studies (visuospatial task [16], executive n-back task [18]) focusing on standing performance and also in the one study addressing walking performance ([104]; working memory task). Effects on ST performance were not reported. Most effects on cognitive performance were reported for GDTs. That is, increased DT cognitive performance were found in three studies while standing and two studies while walking (standing [9, 28, 29]; walking $[66,67])$. Two studies also reported effects on cognitive performance under ST conditions $[29,66]$.

A further aim of this review was to understand the influence of the cognitive task complexity and stimulus-response combinations on performance in motor-cognitive DT situations. To our knowledge, no study systematically compared cognitive tasks of different complexity and variations in stimulus-response settings in motor-cognitive tasks. Only few studies have compared the influence of different task settings before (e.g., [10, 18, 55]). It has been shown that DT costs increase with increasing task demands as a consequence of mentally processing two tasks simultaneously or switching between the tasks (e.g., $[10,55])$. That is, participants might be not able to divide their attentional resources successfully to perform both tasks on a high level. The included studies differed with regard to the complexity and the stimulus-response of the cognitive task (cf. Table 5). The most investigated cognitive task in the eligible studies was controlled processing $(n=5 ;[23,66,67,80,93])$. Three out of this studies reported increased cognitive performance while walking $[66,67,80]$, all of them with a GDT for an 
auditory-verbal task. Two studies which focused on executive control tasks [29: GDT; 18: SDT] reported improved cognitive DT performance for an auditory-verbal [18] and a visual-verbal secondary task [29]. Improvements of processing speed task were found by all of the three authors who investigated auditory-verbal processing speed tasks [9, 28] with GST and GDT. Moreover the SDT of [18] increased the performance of a visual-verbal visuospatial task. In sum, DT training (GDT and SDT) programmes were able to improve all task complexities, whereas the GST programmes only showed increased processing speed, which is the lowest task complexity.

Previous findings of a study by Pellechia [55], where participants had to perform auditory-verbal controlled processing tasks with increasing difficulties (digit reversal, classification, counting backwards by three) while standing, showed increased postural sway with increasing cognitive load. Only two studies covered in this review used cognitive tasks with raising difficulties: the GDT of [80] and the SDT of [18] revealed improvements of executive functions and reduced DT costs while standing [18]. Further studies are needed that use an adaptive design (raising difficulties) and that focus on tasks with a high cognitive load, like executive tasks.

It is also unclear if the modalities of the stimulusresponse influence DT performance as well. Research results illustrated that under specific DT conditions, i.e., when postural stability is necessary to manage the secondary task (visual cognitive tasks), postural sway decreases and stability improves $[74,76]$. Here, the cognitive task is sometimes described as a supra-postural task $[57,74,75]$. Therefore motor performance under DT conditions might benefit from sets that need visual information acquisition, whereas task sets that involve internal interfering factors might lead to a reduced motor-cognitive DT performance $[2,31]$. As reported, a preference for verbal responses can be observed with most studies applying an auditory $(n=8 ;[9,28,66,67$, $80,93])$ or a visual cognitive stimulus $(n=3 ;[16,18,23])$ with a verbal response. One study used an auditory task with a motor response [104]. There were more studies that showed improvements for an auditory-verbal response task but this might be the effect because this was the most used stimulus-response condition.

One might assume that with regard to the reduced capacity hypothesis [32] with increasing cognitive load, less resources are available to manage DT situations and that task managing will be more conflicting if both tasks need the same internal information channel. In this situation, we suppose both tasks have to be processed in one modality and by the use of the same motor coordination processes for response. However, for the small amount of available studies in the current review it remains unclear whether there is an advantage for a specific secondary task or stimulus-response combination. Thus, one can only speculate about the effect of the secondary cognitive task, either the task characteristics or the stimulusresponse mode, on DT performance and we were not able to answer our research questions whether the training effects differ between different complex cognitive tasks/cognitive task with different characteristics. A systematic research on the influence of the task complexity and stimulus-response on DT performance might elucidate the role of cognitive load on adequate resource allocation. We suppose this is needed to conduct appropriate DT training interventions to improve specific DT situations in daily living which include also visual-motor responses like the traffic situation that was described in our introduction. Following this, future training interventions should also consider the stimulus-response mode of the cognitive task and the effect of internal interfering factors.

It is also important to consider whether performance gains can be transferred to other, e.g., everyday tasks. Transfer effects have been only addressed in one study for the cognitive dimension. Silsupadol and co-workers [66] failed to show transfer to a new DT situation using a walking and executive control task. Also cognitive studies - e.g., Owen and colleagues [50], who found cognitive improvements for all trained tasked after a 6-week computerized cognitive training - failed to reveal transfer effects to untrained (even close related) task. Transfer effects in cognitive training studies have only been shown when the training fulfils specific criteria, i.e., variable or randomized task switch training [30]. This might be a reason why, for example, cognitive DT interventions like a driving intervention [13] or the cognitive training reported by $\mathrm{Li}$ et al. [39], both with a variable task prioritization training, revealed transfer to a new DT situations. Also, a flexible resource allocation or prioritisation of a requested task in motor-cognitive DT situations has been shown to improve DT performance (standing [18]; walking [86, 103]). We suppose that variable task prioritization training might help to develop strategies to focus on different tasks and thus promote transfer. For example, when older adults show a tendency to prioritize the (lower-extremity) motor task in DT situations to protect themselves from falls [58], training interventions on motorcognitive DT performance should build up strategies for task prioritization and switching between tasks as described by Silsupadol and co-workers [66, 67] as well as Bherer and co-workers [8] and $\mathrm{Li}$ and colleagues [39]. In line with findings of Segev-Jacubovski and colleagues [60] also our results suggest that multimodality interventions that combine motor and cognitive training should be directed into future DT interventions. They also addressed to use training protocols, to reduce fall risks like done in 
the studies of Silsupadol and colleagues $[66,67]$ or $\mathrm{Li}$ et al. [39].

Overall, motor-cognitive DT training results in healthy older adults are heterogeneous. Most benefits for standing and walking DT conditions seem to be reached by general or specific DT trainings. One has, however, to keep in mind that the specific ST and DT interventions as well as the influence of different task complexities are not examines sufficiently. Therefore the results of this review need to be interpreted carefully, cannot be generalized and do not allow defining a training methodology with greatest effectiveness to improve motor-cognitive physical functioning. To identify the most beneficial intervention that improves motorcognitive DT performance of healthy older adults and help them to manage complex daily life situations, more systematic research is needed.

There are some limitations of this review, which have to be considered. First, there are only six RCTs eligible and the included studies differed in methodological quality (cf. Table 2). Even for the RCTs, we had some methodological issues like validity of the measurements or unclear randomization process. Furthermore, most of the studies did not clearly report their statistics, thus we were not able to gain data for a meta-analysis. For the GDT programmes one have to consider that four studies reported results for both, standing and walking, which might result in a slight reporting bias. Moreover, we identified only a few SST and SDT studies. Therefore the results for these interventions are limited.

\section{Conclusions}

Improvements in motor and cognitive performance in DT situations can be provided with outcome specific and general DT exercises. Results suggest that to improve cognitive and motor performance optimally the training protocols should prefer a DT training over an ST training, include a certain level of exercise load such as rising difficulties, a certain duration and level of task specificity to gain task related adaptations, and variable task prioritization of the training tasks. For practical implications and to provide transfer into everyday situations, the intervention needs to build up rather than general task managing strategies. Future DT training studies should systematically compare of all four training interventions with an RCT, an appropriate sample size, and systematic variations in complexity and stimulus-response conditions of the cognitive task.

Conflict of interest The authors declare that they have no competing interests and there was no funding for this review.
Annex

Table 5 Excluded studies (for Annex)

\begin{tabular}{ll}
\hline Article & $\begin{array}{l}\text { Reason for } \\
\text { exclusion }\end{array}$
\end{tabular}

Bherer L et al. (2008) Transfer effects in taskset cost and dual-task cost after dual-task training in older and younger adults: further evidence for cognitive plasticity in attentional control in late adulthood. Experimental Aging Research 34(3): 188-219

Bherer L, Peterson MS, Kramer AF, Colcombe S, No motor task Erickson K, Becic E (2005) Training effects on standing or dual-task performance: are there age-related differences in plasticity of attentional control? Psychology and Aging. 20(4): 695-709

Bock O (2008) Dual task costs while walking No intervention increase in old age for some, but not for other tasks: an experimental study of healthy young and elderly persons. Journal of

Neuroengineering and Rehabilitation 5: 5-27

Cassavaugh ND, Kramer AF (2009) Transfer of Focused on driving computerbased training to simulated driving performance in older adults. Applied Ergonomics 40: 943952

Doumas M, Smoklers C, Krampe RT (2008) Task No intervention priorization in aging: effects of sensory information on concurrent posture and memory performance. Exp Brain Res 187: 275-281

Fraser SA, Li KZH, Penhune VB (2010) Dual- No pre-post task performance reveals increased involvement of executive control in fine motor sequencing in healthy aging. J Gerontol B Psychol Sci Soc Sci 65(5): 526-535

Hall CD, Heusel-Gillig L (2010) Balance rehabilitation and dual-task ability in older adults. Journal of Clinical Gerontology and Geriatrics 1(1): 22-26

Halvarsson A, Olsson E, Faren E, Pettersson A, Stahle A (2011) Effects of new, individually adjusted, progressive balance group training for elderly people with fear of falling and tend to fall: a randomized controlled trial. Clinical Rehabilitation 25(11): 1021-1031

Laufer Y (2008) Effect of cognitive demand during training on acquisition, retention and transfer of a postural skill. Human Movement Science 27: 126-141

Li KZ et al. (2010) Benefits of cognitive dual- No motor training task training on balance performance in healthy older adults. J Gerontol A Biol Sci Med Sci 65(12): 1344-1352

Lindemann, U., Hammer, W, Muche, R, Nikolaus, Th, Becker, C (2003) postural control in the elderly. Effect of a twelve week Tai Chi-Qigong intervention in healty elderly. EuroJGer. Vol. 5(4):182-186

Maquestiaux F, Hartly AA, Bertsch J (2004) Can practice overcome age-related differences in the psychological refractory period effect? Psychology and Aging 19(4): 649-667
No older adults No control group

No motor task

(n)

\section{Participants used canes or walker \\ Participants were}

$$
\begin{aligned}
& \text { mixed } \\
& \text { between healthy } \\
& \text { and } \\
& \text { unhealthy }
\end{aligned}
$$ \\ No motor task \\ standing or \\ walking
}

No motor task


Table 5 (continued)

\begin{tabular}{ll}
\hline Article & $\begin{array}{l}\text { Reason for } \\
\text { exclusion }\end{array}$ \\
\hline
\end{tabular}

Marmeleira JF, Godinho MB, Fernandes OMT Focused on driving (2009) The effects of an exercise program on performance several abilities associated with driving performance in older adults. Accident Analysis and Prevention 41: 90-97

Marmeleira JFE, de Melo FMS, Godinho MAB Focused on driving (2011) Exercise can improve speed of behavior in older drivers. Journal of Aging and Physical Activity 19(1): 48-61

McDowd JM (1986) The effects of age and extended practice on divided attention performance. Journal of Gerontology 41(6): 764-749

Melzer I, Marx R, Kurz I (2009) Regular exercise in the elderly is effective to preserve the speed of voluntary stepping under singletask condition but not under DT-condition: a case control study. Gerontology 55: 49-57

Nejati V et al. (2008) Dual task interference in implicit sequence learning by young and old adults. International Journal of Geriatric Psychiatry 23: 801-804

Pellecchia GL (2005) Dual-task training reduces impact of cognitive task on postural sway. Journal of Motor Behavior 37(3): 239-246

Shinar D, Tractinsky N, Compton R (2005)

Effects of practice, age, and task demands, on interference from a phone task while driving. Accident Analysis and Prevention 37: 315326

Segev-Jacubovski O, Herman T, YogevSeligmann G, Mirelman A, Giladi N, Hausdorff JM (2011) The interplay between gait, falls and cognition: can cognitive therapy reduce fall risk? Expert Review of

Neurotherapeutics 11 (7): 1057-1075

Simoneau M, Begin F, Teasdale N (2006) The No intervention effects of moderate fatigue on dynamic balance control and attentional demands. J

Neuroengineering Rehabil 3: 22

Silsupadol P. et al. (2006) Training of balance under single- and dual-task conditions in older adults with balance impairment. Physical Therapy 86(2): 269-281

Sparrow W, Begg RK, Parker S (2006) Aging effects on visual reation time in a single task condition and when treadmill walking. Motor Control 10: 201-211

Toulotte C, Thevenon A, Fabre C (2004) Effects French language of training on static and dynamic balance in elderly subjects who have had a fall or not. Annales de Readaptation et de Medecine Physique. 47(9): 604-610

Tsang PS, Shaner TL (1998) Age, attention, expertise, and time sharing performance. Psychology and Aging 13: 323-347

Vaillant J et al. (2006) Balance, aging, and osteoporosis: effects of cognitive exercises combined with physiotherapy. Joint Bone Spine 73: 414-418

Focused on upper extremities

Case control study

No motor task

No elderly

Focused on driving performance

Case reports

No intervention

Focused on flying performance

Focused on osteoporosis
Table 5 (continued)

$\begin{array}{ll}\text { Article } & \begin{array}{l}\text { Reason for } \\ \text { exclusion }\end{array}\end{array}$

Verhoeff LL et al. (2009) Effects of

No pre-post

biofeedback on trunk sway during dual

tasking in the healthy young and elderly.

Gait \& Posture 30: 76-81

Voelcker-Rehage C, Alberts JL (2007) Effect of Focused on upper motor practice on dual-task performance in extremities older adults. Journal of Gerontology:

Psychological Sciences 62B: P141-P148

Wu T, Hallett M (2005) The influence of normal Focused on upper human ageing on automatic movements. J extremities Physiol 562: 605-615

Wu W et al. (2010) The effect of Chinese Yuanji-Dance on dynamicbalance and the associated attentional demands in elderly adults. Journal of Sports Science and Medicine 9: 116-126

Yamada M et al. (2011) Effects of a DVD-based Participants used seated dual-task stepping exercise on fall risk canes or walker factors among community-dwelling elderly adults. Telemedicine Journal \& E-Health 17 (10): 768-772

Yamada M, Aoyama T, Tanaka B, Nagai K, Participants used Ichihashi N (2011) Seated stepping exercise in canes or walker a dual-task condition improves ambulatory function with a secondary task: A randomized controlled trial. Aging - Clinical and

Experimental Research. 23 (5-6): 386-392

Yogev-Seligmann G et al. (2010) How does explicit prioritization alter walking during dual-task performance? Effects of age and sex on gait speed and variability. Physical Therapy 90(2):177-186

\section{References}

1. Albinet C, Bernard PL, Palut Y (2006) Attentional control of postural stability in institutionalised elderly people: effects of a physical exercise program. Ann Readapt Med Phys 49:625631

2. Al-Yahya E et al (2010) Cognitive motor interference while walking: a systematic review and meta-analysis. Neurosci Biobehav Rev 35:715-728

3. Arnold CM, Faulkner RA (2010) The effect of aquatic exercise and education on lowering fall risk in older adults with hip osteoarthritis. J Aging Phys Act 18(3):245-260

4. Baltes PB, Lindenberger U (1997) Emergence of a powerful connection between sensory and cognitive functions across the adult life span: a new window to the study of cognitive aging? Psychol Aging 12(1):12-21

5. Beauchet $\mathrm{O}$ et al (2009) Stops walking when talking: a predictor of falls in older adults? Eur J Neurol 16:786-795

6. Berger L, Bernard-Demanze L (2010) Age-related effects of a memorizing spatial task in the adults and elderly postural control. Gait Posture 2:300-302

7. Bherer L, Peterson MS, Kramer AF, Colcombe S, Erickson K, Becic E (2005) Training effects on dual-task performance: are 
there age-related differences in plasticity of attentional control? Psychol Aging 20(4):695-709

8. Bherer L et al (2008) Transfer effects in task-set cost and dualtask cost after dual-task training in older and younger adults: further evidence for cognitive plasticity in attentional control in late adulthood. Exp Aging Res 34(3):188-219

9. Bisson E et al (2007) Functional balance and dual-task reaction times in older adults are improved by virtual reality and biofeedback training. Cyberpsychol Behav 10:16-23

10. Bock O (2008) Dual task costs while walking increase in old age for some, but not for other tasks: an experimental study of healthy young and elderly persons. J Neuroeng Rehabil 5:5-27

11. Brown SW, Bennett ED (2002) The role of practice and automaticity in temporal and nontemporal dual-task performance. Psychol Res 66(1):80-89

12. Canning CG, Ada L, Woodhouse E (2008) Multiple-task walking training in people with mild to moderate Parkinson's disease: a pilot study. Clin Rehabil 22:226-233

13. Cassavaugh ND, Kramer AF (2009) Transfer of computer-based training to simulated driving in older adults. Appl Ergon 40:943952

14. Colcombe SJ, Kramer AF (2003) Fitness effects on the cognitive function of older adults: a meta-analytic study. Psychol Sci 14(2):125-130

15. Corriveau H, Hébert R, Prince F, Raîche M (2000) Intrasession reliability of the "center of pressure minus center of mass" variable of postural control in the healthy elderly. Arch Phys Med Rehabil 81(1):45-48

16. Dault MC, Frank JS (2004) Does practice modify the relationship between postural control and execution of a secondary task in young and older individuals? Gerontology 50:157-164

17. Doumas M, Rapp MA, Krampe RT (2009) Working memory and postural control: adult age differences in potential for improvement, task priority, and dual tasking. J Gerontol Psychol Sci 64B:193-201

18. Doumas M, Smoklers C, Krampe RT (2008) Task priorization in aging: effects of sensory information on concurrent posture and memory performance. Exp Brain Res 187:275-281

19. Eversheim U, Bock O (2001) Evidence for processing stages in skill acquisition: a dual-task study. Learn Mem 8(4):183-189

20. Faulkner KA et al (2007) Multitasking: association between poorer performance and a history of recurrent falls. JAGS 55:570-576

21. Gillespie LD et al (2007) Interventions for preventing falls in elderly people (review). Cochrane Libr 11:1-289

22. Hall CD, Heusel-Gillig L (2010) Balance rehabilitation and dualtask ability in older adults. J Clin Gerontol Geriatr 1(1):22-26

23. Hall CD, Miszko T, Wolf SL (2009) Effects of Tai Chi intervention on dual-task ability in older adults: a pilot study. Arch Phys Med Rehabil 90(3):525-529

24. Halvarsson A, Olsson E, Faren E, Pettersson A, Stahle A (2011) Effects of new, individually adjusted, progressive balance group training for elderly people with fear of falling and tend to fall: a randomized controlled trial. Clin Rehabil 25(11):1021-1031

25. Hausdorff JM, Yogev G, Springer S, Simon ES, Giladi N (2005) Walking is more like catching than tapping: gait in the elderly as a complex cognitive task. Exp Brain Res 164:541-548

26. Hausdorff JM et al (2008) Dual-task decrements in gait: contributing factors among healthy older adults. J Gerontol A Biol Sci Med Sci 63(12):1335-1343

27. Hedden T, Gabrieli JDE (2004) Insights into the ageing mind: a view from cognitive neuroscience. Nat Rev Neurosci 5:87-97

28. Heiden E, Lajoie Y (2010) Games-based biofeedback training and the attentional demands of balance in older adults. Aging Clin Exp Res 22(5-6):367-373
29. Hiyamazu M, Morioka S, Shomoto K, Shimada T (2011) Effects of dual task balance training on dual task performance in elderly people: a randomized controlled trial. Clin Rehabil 26(1):58-67

30. Jaeggi SM, et al (2008) Improving fluid intelligence with training on working memory. Proc Natl Acad Sci 1-5

31. Jamet $M$ et al (2007) Age-related part taken by attentional cognitive processes in standing postural control in a dual-task context. Gait Posture 25:179-184

32. Kahnemann D (1973) Attention and effort. Prentice Hall, Englewood Cliffs

33. Kramer AF, Hahn S, Gopher D (1999) Task coordination and aging: explorations of executive control processes in the task switching paradigm. Acta Psychol 101:339-378

34. Lafond D, Duarte M, Prince F (2004) Comparison of three methods to estimate the center of mass during balance assessment. J Biomech 37:1421-1426

35. Lajoie Y (2004) Effect of computerized feedback postural training on posture and attentional demands in older adults. Aging Clin Exp Res 16:363-368

36. Latham NK et al (2004) Systematic review of progressive resistance strength training in older adults. J Gerontol Med Sci 59A(1):48-61

37. Laufer Y (2008) Effect of cognitive demand during training on acquisition, retention and transfer of a postural skill. Hum Mov Sci 27:126-141

38. Li KZH, Lindenberger U (2002) Relations between aging sensory/sensorimotor and cognitive functions. Neurosci Biobehav Rev 26:777-783

39. Li KZ, Roudaia E, Lussier M, Bherer L, Leroux A, McKinley PA (2010) Benefits of cognitive dual-task training on balance performance in healthy older adults. J Gerontol A Biol Sci Med Sci 65:1344-1352

40. Lindemann U, Hammer W, Muche R, Nikolaus T, Becker C (2003) Postural control in the elderly. Effect of a twelve week Tai Chi-Qigong intervention in healty elderly. Euro J Ger 5(4):182-186

41. Lövdén $\mathrm{M}$ et al (2008) Walking variability and working-memory load in aging: a dual-process account relating cognitive control to motor control performance. J Gerontol B Psychol Sci Soc Sci 63(3):P121-P128

42. Maquestiaux F, Hartly AA, Bertsch J (2004) Can practice overcome age-related differences in the psychological refractory period effect? Psychol Aging 19(4):649-667

43. Marmeleira JFE, de Melo FMS, Tlemcani M, Godinho MAB (2011) Exercise can improve speed of behavior in older drivers. J Aging Phys Act 19(1):48-61

44. Marmeleira JF, Godinho MB, Fernandes OMT (2009) The effects of an exercise program on several abilities associated with driving performance in older adults. Accid Anal Prev 41:90-97

45. McCafferty WB, Horvath SM (1977) Specificity of exercise and specificity of training: a subcellular review. Res Q 48:358-371

46. McDowd JM (1986) The effects of age and extended practice on divided attention performance. J Gerontol 41(6):764-769

47. Melzer I, Marx R, Kurz I (2009) Regular exercise in the elderly is effective to preserve the speed of voluntary stepping under singletask condition but not under DT-condition: a case control study. Gerontology 55:49-57

48. Navon D, Gopher D (1979) On the economy of the humanprocessing system. Psychol Rev 86:214-255

49. Nejati V et al (2008) Dual task interference in implicit sequence learning by young and old adults. Int J Geriatr Psychiatry 23:801804

50. Owen AM et al (2010) Putting brain training to the test. Nature 465:775-779

51. Park DC, Reuter-Lorenz PA (2009) The adaptive brain: aging and neurocognitive scaffolding. Annu Rev Psychol 60:173-196 
52. Pashler H (1994) Dual-task interference in simple tasks: data and theory. Psychol Bull 116:220-244

53. Pashler H, Johnston JC, Ruthruff E (2001) Attention and performance. Annu Rev Psychol 52:629-651

54. Pellecchia GL (2005) Dual-task training reduces impact of cognitive task on postural sway. J Mot Behav 37(3):239-246

55. Pellechia G (2003) Postural sway increases with attentional demands of concurrent cognitive task. Gait Posture 18:29-34

56. Pichierri G, Wolf P, Murer K, de Bruin ED (2011) Cognitive an cognitive-motor interventions affecting physical function: a systematic review. BMC Geriatr 11(29):1471-2318

57. Riley MA et al (1999) Postural stabilisation for the control of touching. Hum Mov Sci 18:795-817

58. Schäfer S, Schumacher V (2011) The interplay between cognitive and motor functioning in healthy older adults: findings from dualtask studies and suggestions for intervention. Gerontology 57:239246

59. Schwenk M, Zieschang T, Oster P, Hauer K (2010) Dual-task performances can be improved in patients with dementia: a randomized controlled trial. Neurology 74:1961-1968

60. Segev-Jacubovski O, Herman T, Yogev-Seligmann G, Mirelman A, Giladi N, Hausdorff JM (2011) The interplay between gait, falls and cognition: can cognitive therapy reduce fall risk? Expert Rev Neurother 11(7):1057-1075

61. Sherrington C, Whitney C, Lord SR, Herbert RD, Cumming RG, Close JC (2008) Effective exercise for the prevention of falls: a systematic review and meta-analysis. JAGS 56(12):2234-2243

62. Shigematsu R, Okura T, Nakagaichi M, Tanaka K, Sakai T, Kitazumi S, Rantanen T (2008) Square-stepping exercise and fall risk factors in older adults: a single-blind, randomized controlled trial. J Gerontol A Biol Sci Med Sci 63(1):76-82

63. Shigematsu R, Okura T, Sakai T, Rantanen T (2008) Squarestepping exercise versus strength and balance training for fall risk factors. Aging Clin Exp Res 20(1):19-24

64. Shinar D, Tractinsky N, Compton R (2005) Effects of practice, age, and task demands, on interference from a phone task while driving. Accid Anal Prev 37:315-326

65. Silsupadol P, Siu KC, Shumway-Cook A, Woollacott MH (2006) Training of balance under single- and dual-task conditions in older adults with balance impairment. Phys Ther 86(2):269-281

66. Silsupadol P et al (2009) Effects of single-task versus dual-task training on balance performance in older adults: a double-blind, randomized controlled trial. Arch Phys Med Rehabil 90:381-387

67. Silsupadol P et al (2009) Training-related changes in dual-task walking performance of elderly persons with balance impairment: a double-blind, randomized controlled trial. Gait Posture 29:634 639

68. Simoneau M, Begin F, Teasdale N (2006) The effects of moderate fatigue on dynamic balance control and attentional demands. J Neuroengineering Rehabil 3:22

69. Singh MAF (2002) Exercise comes of age: rationale and recommendations for a geriatric exercise prescription. J Gerontol Biol Sci Med Sci 57(5):M262-M282

70. Siu KC et al (2008) Dual-task interference during obstacle clearance in healthy and balance-impaired older adults. Aging Clin Exp Res 20(4):349-354

71. Sparrow W, Begg RK, Parker S (2006) Aging effects on visual reaction time in a single task condition and when treadmill walking. Mot Control 10:201-211

72. Springer S et al (2006) Dual-tasking effects on gait variability: the role of aging, falls and executive function. Mov Disord 21(7):950-957

73. Srygley JM et al (2009) When does walking alter thinking? Age and task associated findings. Brain Res 9(1253):92-99

74. Stoffregen TA et al (1999) Postural stabilisation of looking. J Exp Psychol Hum Percept Perform 25(6):1641-1658
75. Stoffregen TA et al (2000) Modulation postural control to facilitate visual performance. Hum Mov Sci 19:203-220

76. Swan L et al (2004) Improving balance by performing a secondary cognitive task. Br J Psychol 95(Pt 1):31-40

77. Swanenburg J, de Bruin ED, Favero K, Uebelhart D, Mulder T (2008) The reliability of postural balance measures in single and dual tasking in elderly fallers and non-fallers. BMC Musculoskelet Disord 9:1-10

78. Toulotte C, Thevenon A, Fabre C (2004) Effects of training on static and dynamic balance in elderly subjects who have had a fall or not. Ann Readapt Med Phys 47(9):604-610

79. Toulotte C, Thevenon A, Fabre C (2006) Effects of training and detraining on the static and dynamic balance in elderly fallers and non-fallers: a pilot study. Disabil Rehabil 28:125-133

80. Trombetti A, Hars M, Herrmann FR, Kressig RW, Ferrari S, Rizzoli R (2011) Effect of music-based multitask training on gait, balance, and fall risk in elderly people: a randomized controlled trial. Arch Intern Med 171(6):525-533

81. Tsang PS, Shaner TL (1998) Age, attention, expertise, and time sharing performance. Psychol Aging 13:323-347

82. Vaillant J et al (2006) Balance, aging, and osteoporosis: effects of cognitive exercises combined with physiotherapy. Joint Bone Spine 73:414-418

83. van Tulder M, Furlan A, Bombardier C, Bouter L (2003) Updated method guidelines for systematic reviews in the Cochrane collaboration back review group. Spine 28(12):1290-1299

84. Van Uden CJT, Besser MP (2004) Test-retest reliability of temporal an spatial gait characteristics measured with an instrumented walkway system (GAITRite ${ }^{\circledR}$ ). BMC Musculoskelet Disord 5(13):1-4

85. Verghese J, Mahoney J, Ambrose AF, Wang C, Holtzer R (2010) Effect of cognitive remediation on gait in sedentary seniors. $J$ Gerontol A Biol Sci Med Sci 65:1338-1343

86. Verghese $\mathrm{J}$ et al (2006) Walking while talking: effect of task prioritization in the elderly. Arch Phys Med Rehabil 88:50-53

87. Verhaeghen P, Cerella J (2002) Aging, executive control, and attention: a review of meta-analyses. Neurosci Biobehav Rev 26:849-857

88. Verhaeghen P et al (2003) Aging and dual-task performance: a meta-analysis. Psychol Aging 18(3):443-460

89. Verhoeff LL et al (2009) Effects of biofeedback on trunk sway during dual tasking in the healthy young and elderly. Gait Posture 30:76-81

90. Voelcker-Rehage C (2008) Motor-skill learning in older adults a review of studies on age-related differences. Eur Rev Aging Phys Act 5:5-16

91. Voelcker-Rehage C, Alberts JL (2007) Effect of motor practice on dual-task performance in older adults. J Gerontol Psychol Sci 62B:P141-P148

92. Welford AT (1967) Single-channel operation in the brain. Acta Psychol 27:5-22

93. Westlake KP, Culham EG (2007) Sensory-specific balance training in older adults: effect on proprioceptive reintegration and cognitive demands. Phys Ther 87:1274-1283

94. Wickens CD (1980) The structure of attentional resources. In: Nickerson R (ed) Attention and performance VIII. Erlbaum, Hillsdale, pp 239-257

95. Wickens CD (2002) Multiple resource and performance prediction. Theor Issues Ergon Sci 3:159-177

96. Woollacott M, Shumway-Cook A (2002) Attention and the control of posture and gait: a review of an emerging area of research. Gait Posture 16:1-14

97. Wu T, Hallett M (2005) The influence of normal human ageing on automatic movements. J Physiol 562:605-615

98. Wu W et al (2010) The effect of Chinese Yuanji-Dance on dynamic balance and the associated attentional demands in elderly adults. J Sports Sci Med 9:116-126 
99. Yamada M, Aoyama T, Tanaka B, Nagai K, Ichihashi N (2011) Seated stepping exercise in a dual-task condition improves ambulatory function with a secondary task: a randomized controlled trial. Aging Clin Exp Res 23(5-6):386-392

100. Yamada $\mathrm{M}$ et al (2011) Effects of a DVD-based seated dual-task stepping exercise on the fall risk factors among communitydwelling elderly adults. Telemed J E Health 17(10):768-772

101. Yang YR, Wang RY, Chen YC, Kao MJ (2007) Dual-task exercise improves walking ability in chronic stroke: a randomized controlled trial. Arch Phys Med Rehabil 88:1236-1240
102. Yogev-Seligman G, Giladi N, Brozgol M, Hausdorff JM. (2011) A training program to improve gait while dual tasking in patients with Parkinson's disease: a pilot study. Arch Phys Med Rehabil 93(1):176-181

103. Yogev-Seligmann G et al (2010) How does explicit prioritization alter walking during dual-task performance? Effects of age and sex on gait speed and variability. Phys Ther 90(2):177-186

104. You JH et al (2009) Effects of dual-task cognitive-gait intervention on memory and gait dynamics in older adults with a history of falls: a preliminary investigation. NeuroRehabilitation 24:193-198 